\author{
CASES \\ ILLUSTRATIVE OF

\section{THE PATHOLOGY OF THE EAR.} \\ BY \\ JAMES HINTON, M.R.C.S. \\ COMMUNICATED BY \\ EDWARD STANLEY, F.R.S.
}

Received Feb. 2d, 1856.-Read Feb. 12tl, 1856.

The series of investigations into the pathology of the ear, laid before the Royal Medical and Chirurgical Society by Mr. Toynbee, have done much to remove the ignorance which previously existed with regard to the diseases of that organ. But the subject is of such extent, and, until lately, has been so little studied, that there is still room for additions to our knowledge. I am therefore induced to bring under the notice of the Society the results of 56 dissections of the ear, made by myself.

The general results of these dissections go to confirm the views advanced by Mr. Toynbee, to whom I am greatly indebted for permission to examine and compare the specimens contained in his museum. A few of them seem to warrant more particular mention.

Of the 56 ears, 12 were healthy. Of the remaining 44 which deviate more or less from the healthy standard, 7 belonged to persons known to be deaf; 9 belonged to persons 
known not to be deaf; that is, in whom the power of hearing the voice was observed to be unimpaired, so far as could be judged, to the time of death or loss of consciousness. Of the 28 of which the histories are unknown, 15 present considerable evidences of old-standing disease, and 13 are more slightly affected.

I shall first relate briefly the history of some of the cases; and afterwards present a summary of the morbid appearances detected.

Case 1.-Deposits, probably tubercular, in either tympanum; caries of the petrous bone on the left side; abscess in the left cerebral hemisphere; erysipelas of the scalp.

J- R-, æt. 19, was admitted into St. Bartholomew's Hospital on the 26th April, 1855. His mother states, that at two years of age he fell upon his head, from a height of two feet. This fall was followed by an illness, during which lumps formed below both ears, and a discharge issued from the left. From that time he has been more or less deaf, and has complained of pain in the left ear, which has been more severe whenever the discharge was not present. On account of his dulness, his father had been much in the habit of boxing his ears. For about a fortnight previously he had suffered from a peculiar shooting pain around the head. On admission into the hospital, the entire scalp was in a state of erysipelatous inflammation, and with it there existed great debility, a small feeble pulse, and profuse diarrhœa. After a few days, suppuration was established beneath the occipito frontalis, and openings having been made at various points, the bones were found to be extensively denuded, being covered by the brawny unadherent scalp. There were no acute cerebral symptoms, but he continued to complain of severe frontal headache. About the 12th of May the discharge from the ear reappeared, having previously been absent for more than a month. The suppuration from the scalp continued, and he was greatly weakened 
by occasional attacks of diarrhœa. On the 20th May the headache became more severe, and continued so, but without any fresh symptoms presenting themselves, until he became comatose, about three hours before his death, on the 25th.

Post-mortem examination. - The occipital and the temporal and parietal bones were bare to a great extent, and covered with pus; their surface was dotted over with superficial excavations, giving it a honeycomb appearance. The base of these excavations was rough, and of a pink colour, and many of them were filled with granulations.

The arachnoid was of a dull-yellowish colour, with soft, almost purulent lymph, effused along the track of the principal vessels of the pia mater. The cerebral convolutions on the left side were much flattened. There was a large amount of pus at the base of the brain, and the pia mater, as it dipped in among the convolutions, was covered with thick, puriform fluid, and a similar fluid occupied the anterior cornu of the ventricles, the walls of which were of a bright pink colour, and contrasted with the other parts of the cerebrum, which was, for the most part, unusually pale, as were also the choroid plexuses.

The arachnoid corresponding to that portion of the left hemisphere which rests upon the petrous portion of the temporal bone, bulged before a collection of laudable pus. In all, about two ounces of this fluid occupied, to the destruction of the natural tissue, the middle of this hemisphere, and were contained by a limiting wall of thickened and indurated tissue, highly vascular, vessels being conspicuous running through it in every direction, and an abundance of large exudation cells mixed with filamentous tissue mingling with the constituents of the nervous structure. Thus altered and inflamed, this wall surrounded for a depth of one inch the matter mentioned above; beyond it, for a considerable extent, the cerebrum was quite soft and diffluent.

Both lungs contained numerous groups of small, grey tubercles, the tissue immediately surrounding which was 
vascular in excess. The other thoracic and abdominal organs were healthy.

Examination of the petrous bones.-On the left side, the plate of bone forming the roof of the tympanum is of a dirty-brown colour, thin, easily breaking down, and surrounded, in the recent state, by a line of a bright pink colour. The dura mater covering it was separated from the bone, the discoloration of which appeared through it, but it retained its smooth, polished surface. The tympanic cavity, and upper portion of mastoid cells, are full of a soft, greenish-yellow deposit, of the consistence of putty, mingled with some thick puriform fluid, and it communicates with the external surface of the temporal bone by means of a small aperture between the external meatus and the mastoid process. In the situation of this orifice there is a depression in the bone which might contain a bean, and on both the external and internal surfaces of the bone there are irregular elevated patches, arising from the deposition of new bone. The membrana tympani is greatly thickened, and covered with soft, warty growths, so that its natural structure is completely lost. In the upper part, above the head of the malleus, there is a small oval orifice, about two lines in its greatest length. The dermoid layer of the external meatus is much inflamed and thickened.

The right tympanum contains a deposit firmer than that on the left side, and of a dirty-white colour. It occupies the space between the proper cavity of the tympanum and the mastoid cells, and adheres, by many thread-like bands, to the external osseous wall. The fibrous layers of the membrana tympani are thick and opaque; the membrane is extremely concave, its internal surface being nearly in contact with the inner wall of the tympanum.

The general features of this case are such as commonly arise from abscess in the cerebrum, dependent on caries of the petrous bone, but the peculiar direction taken by the ulcerative action, passing through the external plate of the temporal bone, above the mastoid process, is indicative of 
disease commencing within the mastoid cells in early life; which the history confirms. Mr. Toynbee has pointed out, that during infancy this plate of bone is exceedingly thin, and forms the external boundary of the horizontal portion of the mastoid cells, which is the only portion then developed. In the left ear, which obviously presents an early stage of the same disease, the morbid deposit occupies precisely that position. The deposition of new bone indicates an effort of nature to repair the injury sustained, an effort rendered abortive by the continuance of the disease in the tympanic cavity, causing caries of the superior wall of the tympanum.

For the history of this case, and the opportunity of examining the temporal bones, I am indebted to $\mathrm{Mr}$. Callender.

An early stage of a disease similar to the foregoing, is presented by the following case.

CASE 2.-Tubercular disease of the brain and lungs; the left tympanum containing semi-purulent mucus, the right tympanum containing a small mass of tubercular deposit.

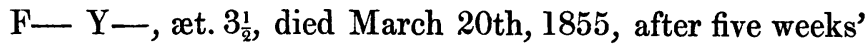
illness, of which the prominent symptoms were fever, with obstinate constipation, and diminution of urine, followed by vomiting, pain in the left side of the head, convulsions, and attacks of syncope. On post-mortem examination, the following appearances were found.

The sinuses and membranes of the brain contained a normal amount of blood. On the left side of the middle lobe of the cerebrum, immediately above the fissure of Silvius, the arachnoid contained numerous small scattered masses of white deposit. These masses covered a space of about three inches vertically by an inch and a half laterally, and appeared nearly to follow the course of the middle cerebral artery. Imbedded in the substance of the left thalamus opticus, immediately beneath this external deposit, was a firm mass of 
pale red colour, about the size of a horse-bean, and surrounded by a large plexus of distended blood-vessels.

Throughout the whole cerebrum, but chiefly in the posterior lobes, were scattered small, firm, red masses of deposit, easily turned out from the surrounding cerebral tissue. There were a few in the pons, several in the crura cerebri; none were found in the cerebellum, which was very pale as compared with the cerebrum, but appeared healthy.

Both lungs were thickly studded with tubercles, the kidneys were large and pale. The mesenteric glands were enlarged, and contained tubercular matter.

Examination of the Temporal Bones. Right side.-The membrana tympani was opaque and vascular; the mucous layer much thickened. Around the heads of the malleus and incus there was a soft deposit of pale yellow colour, apparently about three grains in weight. The mucous membrane lining the tympanum and mastoid cells was ecchymosed; the mastoid cells contained a broad false membrane. Red vessels were seen ramifying on the internal surface of the vestibule.

Left side.-Membrana tympani : dermoid layer more vascular than natural; mucous layer thick. The cavity of the tympanum contained puriform mucus ; the mastoid cells were filled with a dark-coloured viscid fluid, which appeared to discolour the bone above, and contained also a large red false membrane. Membranous bands also united the incus and stapes together. The mucous membrane lining the tympanum and Eustachian tube was very red and thick. The fluid contained in the tympanum consisted of granular matter mixed with numerous pus cells.

In this case the hearing was very acute during the last illness, and the power of distinguishing the voice appeared to be perfectly preserved to the last. The chief disease within the cranium was found in the immediate neighbourhood of the left petrous bone, and it appears at least possible that irritation, propagated from the inflamed tympanum, 
might have acted powerfully as an exciting cause of the fatal tuberculosis of the brain.

Case 3.-Eustachian tubes obstructed by thickening of their lining membrane. Cavity of the tympanum on each side containing purulent mucus.

Samuel H-, æt. $3 \frac{1}{2}$, a well-formed child, of dark complexion, was first seen on the evening of 26th May, 1855. He was then in strong convulsions, affecting nearly all the muscles of the body, and resembling an extremely severe form of chorea. He did not appear to be in pain; there was no heat of skin, or excessive quickness of the pulse; he took fluids when desired. He continued convulsed during the whole night, and died exhausted about 9 o'clock on the following morning.

His history, so far as it could be ascertained, was as follows: He was always a very passionate boy, especially during the few last months of his life. He had enjoyed general good health, never complained of headache, but often seemed more unwilling than other children to have his head touched; he also had a habit of putting his fingers in his ears, but never complained of pain in them, nor had there been any discharge from them. Four or five times he has been noticed to throw himself upon the ground, and roll about, apparently in play, yet so strangely as to attract particular attention. Has been deaf, when he has had a cold, for some time. About three days before his death he became much more deaf than ever before, and so continued.

He had appeared in his usual health until the 24th, three days before his death, when he seemed to be languid and ill. On the evening of that day he fell, and struck his head against the door, but not violently, and apparently quite recovered from the effects of the blow. The next day he was feverish, and laid his head upon the pillow. He first became convulsed on the evening of the $26 \mathrm{th}$, and died in fourteen hours afterwards. 
Examination twenty-four hours after death.-Appearance of body natural. The sac of the arachnoid contained three or four drachms of clear fluid, and the membranes of the brain generally were much congested. No disease was detected in the substance of the brain, which appeared quite healthy throughout. The spinal cord was not examined. The heart was loosely contracted. A small triangular mass of soft lymph, attached by its base to the apex of the heart, lay in the pericardial sac. There were a few dull white patches on the opposed surfaces of the pericardium, which, however, contained no fluid. Structure of the heart healthy.

The lower lobe of the left lung contained scarcely any air ; it did not collapse when the chest was opened, nor crepitate beneath the finger. It was of a dark reddish hue when cut into, and broke down on firm pressure. Portions of it sank in water.

Examination of temporal bones.-The mucous membrane of the fauces around the orifices of the Eustachian tubes was very greatly swollen, and infiltrated with muco-purulent fluid, which exuded from it in great quantity when it was pressed. The Eustachian tubes were closed, by approximation of the thickened lining membrane, from the faucial extremity to within half an inch of the tympanic opening. The cavity of the tympanum on each side contained a redcoloured viscid fluid; and the mucous membrane was red, thick, and velvety. The membranæ tympani were fallen in towards the promontory; they were very vascular, and the mucous layer thick.

On the right side, there were many membranous bands uniting the membrana tympani and all the ossicula to the inner wall of the tympanum; these bands existed also in the mastoid cells, and contained many spots of ecchymosis. The cochlea, also (on the right side), was much congested, and contained a red fluid; and red vessels were seen ramifying on the walls of the vestibule and canals.

The starting point of the morbid condition of the ears, in this case, appears to have been the disease of the faucial 
mucous membrane extending into the tympanum, and causing obstruction of the Eustachian tubes. Hence arose the accumulation of viscid mucus, and the collapse of the membranæ tympani. This latter condition appears to be an almost invariable result of prolonged obstruction of the Eustachian tubes; as if the want of a renewed supply of air within the tympanum caused the membrane to yield to the pressure of the external atmosphere. How far the morbid condition of the tympana, and on the right side of the cochlea also, might have contributed to induce the convulsive seizure during which the patient died, I have no means of forming an opinion.

The other children of this family, two in number, suffer under deafness, which appears to arise from a similar condition of the throat.

In some cases, it appears that irritation, propagated from an inflamed tympanum, gives rise to cerebral disorder of sufficient intensity to destroy life, without any organic lesion discoverable after death. The following was probably a case of this nature.

CASE 4.-A middle aged man was admitted into St. Bartholomew's Hospital in July, 1854, delirious, and in a moribund condition. The particulars of his history are unknown. On examination after death no disease was found, except that the tympanic cavity and mastoid cells on the left side were full of dark coloured unhealthy pus. The membrana tympani was perforated. The layer of bone forming the roof of the tympanum was dark coloured, and the surrounding cancellous structure infiltrated with pus. The dura mater and brain were healthy. On the right side the lower portion of the mastoid cells and the neighbouring bone were dark coloured, and infiltrated with sanguineous fluid. 
Case 5.-Accumulation of mucus in the tympanic cavity; thickening of the mucous membrane.

Harriet B-, æt. 2 years. Died on 27th January, 1855, from exhaustion, after repeated attacks of bronchitis, accompanied with dilatation and hypertrophy of the heart.

Examination of the temporal bones. Right side.-The dermoid layer of the membrana tympani was more vascular than natural. The mucous membrane of the tympanum was slightly reddened and thick. Firm membranous bands united the incus and stapes to the posterior wall of the tympanum. Red vessels were seen ramifying on the surface of the cochlea and semicircular canals.

Left side.-The membrana tympani was more concave than natural, opaque, of a dull leaden mottled aspect from thickening of its mucous layer. It was covered with red vessels converging from the circumference towards the centre. The tympanum and mastoid cells were filled with mingled purulent fluid and viscid mucus. Membranous bands surrounded the crura of the stapes, and united the incus to the chorda tympani nerve. The mucous membrane was red, velvety, and so thick as greatly to diminish the size of the tympanic cavity, and the diameter of the Eustachian tube. It was also very loosely connected with the bony walls of the tympanum, remaining in its position when the bone was removed. The bone itself, however, was healthy. It would seem probable, that in some cases of caries of the walls of the tympanum, arising from inflammation within its cavity, the cause of the loss of vitality of the bone may be rather a deficient supply of blood, owing to detachment of the inflamed mucous membrane, than a direct extension of the inflammatory action.

In this case no impairment of the hearing had been observed by the parents ; but it appeared, on inquiry, that the child had for some time been in the habit of putting 
her hands to her ears, and the mother had frequently picked them with a pin, which seemed to give her pleasure.

CASE 6.-Deafness on the left side. Membranous bands in either tympanum; ossicula on the left side drawn together, and less moveable than natural.

H. W-, a tall, apparently robust man, died suddenly at the age of 52. The muscular structure of the heart was in a state of degeneration.

History. - He suffered from ear-ache in early life, and was noticed to be slightly deaf at about 26 years of age. For some years past he had been unable to hear without great difficulty with the left ear, and when spoken to would habitually turn the right ear. He was able, however, to carry on ordinary conversation with perfect facility, the hearing on the right side being very good. He had always been subject to headache, and three years ago had an illness pronounced to be congestion of the brain.

His mother became deaf in both ears at 7 years of age from scarlatina, and her deafness increased as she advanced in life. All his brothers and sisters, of whom there were four, suffercd in childhood from pain in the ears. His sister, aged 40, is very deaf, the symptoms indicating an affection of the auditory nerve. His two brothers are deaf in a less degree.

Examination of the petrous bones.-The left ear, in which the hearing was impaired, presented the following appearances: The membrana tympani was slightly opaque, thicker and more concave than natural, and rigid. The mucous membrane of the tympanum was red and thick. The ossicula were approximated to each other, and firmly bound by membranous bands, uniting the long crus of the incus to the membrana tympani and to the handle of the malleus, and the stapes to the other ossicles and tympanic walls. The stapes was firmly fixed in the fenestra ovalis, 
being immoveable without considerable pressure. The vestibule and semicircular canals were healthy. The cochlea more vascular than natural. The membrana fenestræ rotundæ was opaque.

Right ear.-The meatus externus contained a small accumulation of soft cerumen. The membrana tympani and mucous membrane of tympanum healthy. The ossicula were surrounded by membranous bands, connecting the membrana tympani and malleus to the long process of the incus, and the stapes and incus to the posterior wall of the tympanum. The ossicles retained their natural mobility. The internal ear was healthy, except a slight vascularity of the cochlea and adjacent part of the vestibule.

Adhesions of the ossicula to each other and to the walls of the tympanum are forms of disease of such frequent occurrence, that great interest attaches to any facts that can throw light upon the influence they exert upon the faculty of hearing. In the foregoing case they exist to about an equal extent on each side; but with this important difference, that in the ear of which the hearing was impaired, the chain of bones is drawn together and rendered immoveable. In the other ear the ossicula retain their proper positions and natural mobility. This case, therefore, affords evidence that membranous bands within the tympanum do not interfere with audition unless they impede the motions of the ossicula, or cause the stapes to press upon the fluid of the vestibule.

In the following case it is probable that the contraction of membranous bands in- the tympanum diminishing the mobility of the ossicula, and especially of the stapes, was a cause of deafness.

Case 7.-A female aged 8 years. Died from dropsy after scarlatina. Was observed to be hard of hearing during her last illness.

Examination of temporal bones.- On each side the meatus 
externus contained desquamating epidermis. The membrana tympani is healthy in structure; but more concave than natural. All the ossicula are united to one another and to the walls of the tympanum by their transparent bands. The heads of the malleus and incus covered with red vessels. Stapes less moveable than natural. Internal ear healthy.

Mucus accumulated within the cavities of the ear will sometimes dry up after death into membrane-like films, which present a very similar appearance to adhesions formed during life. This was observed in the following two cases :

CASE 8.-E. F-, male, at. 17. Died, after eight months' illness, of phthisis complicated with mania. His hearing appeared good to the time of death.

Examination of temporal bones.-The membrana tympani on the right side was very concave, its inner surface being nearly in contact with the promontory. It was covered with thickened epidermis, and the circular fibrous layer was also opaque and thickened to a slight extent. The left membrana tympani was more concave than natural; but its structure was healthy. The cavity of the tympanum on each side contained numerous fine bands uniting the ossicula to each other and to the posterior osseous wall. Broad bands existed also in the mastoid cells. The mucous membrane of the tympanum and the internal ear on each side were healthy. Much thick mucus was collected about the upper part of the pharynx, and the faucial extremity of the Eustachian tubes was filled with transparent viscid mucus. This mucus, having been suffered to remain in the Eustachian tube of the left side, had dried into fine transparent bands, stretching across the tube (which had been laid open), and precisely resembling the bands which existed within the tympanum. 
Case 9.-Ann D-, at. 2. Died from hooping cough complicated with diarrhoea. Hearing not impaired.

Examination of the temporal bones. On the right side the mucous membrane of the tympanum was much congested, of a pinkish hue, and covered with a thin layer of mucus. The cochlea also was congested.

On the left side the epidermis covering the membrana tympani was thick; numerous red ressels ramified in the dermoid layer. The mucous membrane of the tympanum was of a bright red colour, the ossicula were covered with red vessels, and the long process of the incus appeared of a bright pink hue through the membrana tympani. The base of the stapes in each ear formed a strong contrast, by its red colour, to the other parts of the wall of the vestibule. Stretching across the mastoid cells on the left side was a layer of semifluid tenacious substance, of a dirty white colour, which was converted, in the course of a few days, into a firm transparent pseudo-membrane containing red streaks, and closely resembling the membranous bands so frequently found in the same situation.

The tympanum being a cavity containing air which is frequently renewed, it is conceivable that the drying up of mucus during life might give rise to one form of the membranous adhesions which constitute so frequent a pathological appearance.

Examined beneath the microscope the dried mucus presented an agglomeration of granular matter and shrivelled cells of varying thickness and tenacity. A portion of some firm adhesions formed during life presented a distinctly fibrous structure.

Membranous adhesions in the tympanum are not only extremely common, being present in 29 out of 56 dissections, but they are met with also in very early life. One of the specimens exhibited was the right temporal bone of a child, aged 4 months, who died with symptoms of secondary sup- 
puration. It exhibited a broad transparent band uniting the posterior crus of the stapes to the canal of the portio dura nerve.

The following is a short statement of the morbid conditions found in the 44 diseased ears.

I. Eight ears belonging to persons known to be deaf.

Meatus Externus.

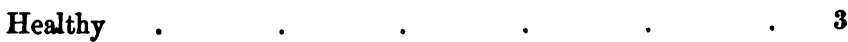

Containing desquamating epidermis $\quad$. $\quad . \quad 3$

Dermoid layer inflamed, and thickened from chronic inflam-

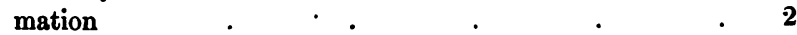

Membrana Tympani.

Concave, and less moveable than natural a $\quad$. $\quad$. $\quad 2$

Concave, opaque, rigid, mucous layer thickened . . . 1

Collapsed, vascular, mucous layer thickened $\quad$. $\quad$. 2

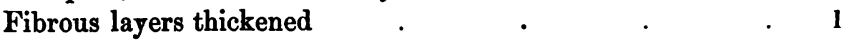

Perforated and thickened $\quad . \quad$. $\quad . \quad . \quad 1$

Perforated, and covered with fungoid granulations . $\quad$. 1

\section{Cavity of the Trmpanum.}

Mucous membrane healthy, containing membranous bands . 2

Mucous membrane red and thick, containing membranous bands

Mucous membrane red and thick, containing membranous

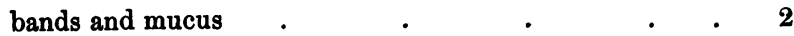

Mucous membrane red and thick, containing pus . $\quad$. 1

Mucous membrane red and ecchymosed, containing scrofulous

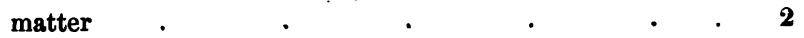

\section{Mastoid Cerus.}

Healthy

Mucous membrane congested $\quad . \quad$. $\quad$. $\quad$. 3

Containing bands and ecchymosis $\quad . \quad$. $\quad . \quad 1$

Containing pus 


\section{Ossicula.}

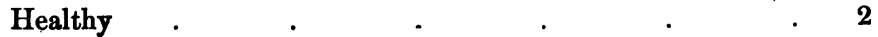

Membrane covering them red $\quad . \quad$. $\quad$. $\quad . \quad 1$

United by membranous bands $\quad$. $\quad$. 4

Imbedded in scrofulous matter and pus $\quad$ r $\quad . \quad$. 2

Stapes less mobile than natural $\quad$ r $\quad$. 2

Eustachian Tube.

Closed by thickened mucous membrane $\quad$ r $\quad$. 2

\section{Cochlea.}

Congested

$\begin{array}{lllll}\text { Congested, and containing red fluid } & \text { - } & \text {. } & & 1\end{array}$

Vestibule and semicircular Canals.

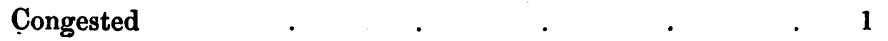

Membrana Fenestre Rotunde.

Opaque

II. Nine ears belonging to persons known not to be deaf.

Meatus Externus.

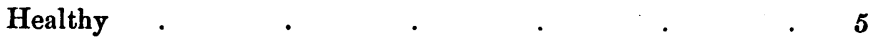

Containing desquamating epidermis $\quad$. $\quad . \quad$. 1

$\begin{array}{lllllllll}\text { Containing cerumen and epidermis } & \text {. } & & & & \end{array}$

\section{Mgmbrana Trmpani.}

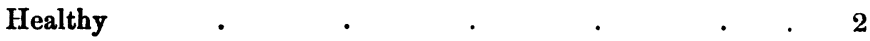

$\begin{array}{llllllllll}\text { Epidermoid layer thick } & \text { - } & & & & & & & & \end{array}$

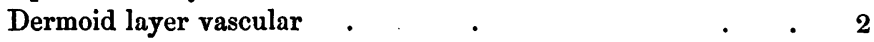

Circular fibrous layer thickened $\quad$. $\quad$. $\quad$. 1

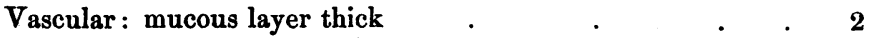

Caviry of the Trmpanum.

Mucous membrane healthy; containing membranous bands . 4

Mucous membrane congested . $\quad$. $\quad$. $\quad$. 2

Mucous membrane thick and red $\quad . \quad$. $\quad$. $\quad . \quad 1$

Mucous membrane thick and red; containing mucus, and membranous bands $\quad$. $\quad$. $\quad$. $\quad$ l 1

Mucous membrane red and ecchymosed; containing scrofulous matter 
Mastoid Cells.

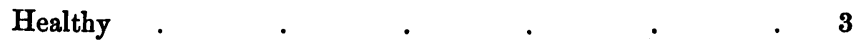

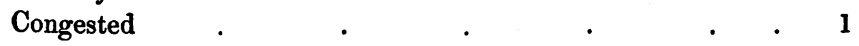

Congested, and containing membranous bands . 2

Healthy, and containing membranous bands . $\quad$. 3

Eustachian Tube.

Small, mucous membrane thick $\quad$. $\quad$. $\quad$. 2

\begin{tabular}{lllllll} 
Containing viscid mucus & . & & & & & \\
\hline & & &
\end{tabular}

Vestibure.

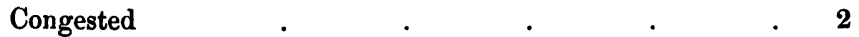

Cochlea.

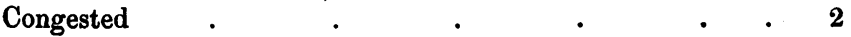

III. Twenty-seven ears; the histories unknown.

\section{Meatus Externus.}

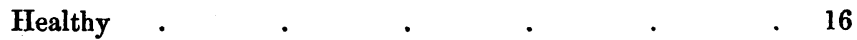

- Containing cerumen $\quad$. $\quad$. $\quad$. $\quad$. 2

Containing cerumen, and thickened epidermis . $\quad 3$

Containing cerumen; dermoid layer red . $\quad$. 3

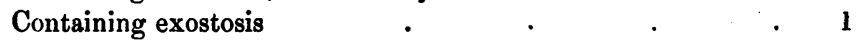

Lower bony wall deficient $\quad \cdot \quad \cdot \quad \cdot \quad \cdot \quad \cdot \quad 1$

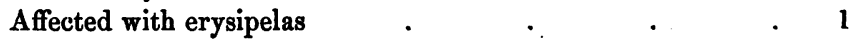

\section{Membrana Trmpant.}

Healthy $\quad . \quad$. $\quad$.

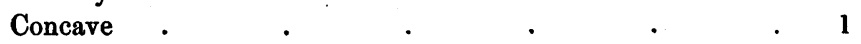

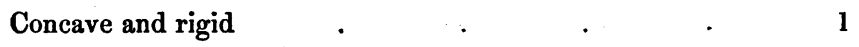

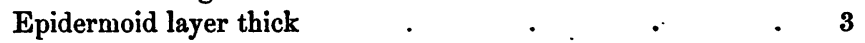

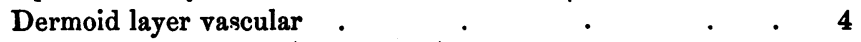

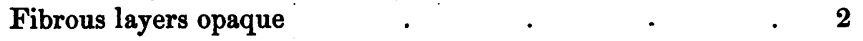

Opaque, relaxed, pressed in by cerumen $\quad \cdot \quad$. $\quad$. 2

Relaxed, concave, dull $\quad$. $\quad$. $\quad$. $\quad$. 1

Drawn in, and adherent to incus . $\quad . \quad$. $\quad$. 1

Opaque and mottled, from thickened mucous layer . $\quad$. 1

Containing calcareous deposit $\quad$. $\quad$ - $\quad$. $\quad$. 1

Containing calcareous deposit, and perforated $\quad$. 2 .

Perforated, the edges of the orifice attached to the incus and

walls of tympanum . $\quad 1$ 


\section{Cavity or the Trmpanum.}

Healthy $\quad$ - $\quad$. $\quad$. $4 \quad$. 4

Mucous membrane congested $\quad$. $\quad$. $\quad$. $\quad$. 1

Mucous membrane congested and thickened . 4

$\begin{array}{llllllllllll}\text { Containing adhesions } & \text {. } & & & & & \end{array}$

Mucous membrane thickened, containing adhesions 4

Mucous membrane thick and red, containing adhesions . 2

Containing red lymph and adhesions $\quad$. $\quad$. $\quad$. 1

Containing a sanguineous fluid and adhesions $\quad$. 3

Containing pus and adhesions, mucous membrane thick and red

\section{Mastoid Cells.}

Healthy

Congested

Containing adhesions

Containing mucus

Containing pus

Sanguineous fluid effused in the cancellous tissue around tympanum (typhus fever) . $\quad$. $\quad$. 2

Bone around the mastoid process infiltrated with fluid and of . dark colour

\section{Ossicura.}

Healthy

United by adhesions. $\quad . \quad 4 \quad . \quad 18$

Red vessels ramifying over them $\quad$. $\quad$. $\quad 5$

Ditto and ecchymosis $\quad . \quad$. $\quad . \quad$. $\quad . \quad 1$

Less mobile than natural $\quad$. $\quad$. $\quad$. $\quad$. 1

Deposit of red lymph on base of stapes . $\quad$ - $\quad$. $\quad$. 1

Malleus partially detached from membrana tympani . 1

Stapes united by membranous ankylosis to margin of fenestra ovalis .

Stapes dislocated into vestibule at posterior border $\quad 1$

\section{Eustachian Tube.}

Very large

Contracted by approximation of bony walls

Thin spiculæ of bone growing from its floor $\quad . \quad+\quad . \quad 1$

Mucous membrane thick and red, containing mucus . 1

Closed by thickened mucous membrane . . . 1 
Cochrua.

Congested

Congested, the contained fluid of a red colour

1

Lamina spiralis broken down: the scalæ towards their termination containing a granular fibrinous exudation, covering the fenestra rotunda

Semicircular Canals.

Red vessels ramifying on their surface $\quad$. $\quad$. $\quad$. 2

Membrane of Fenestra Rotunda.

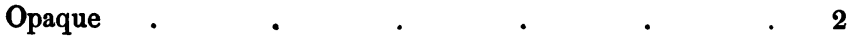

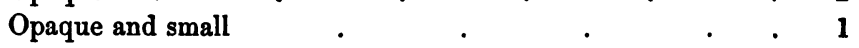

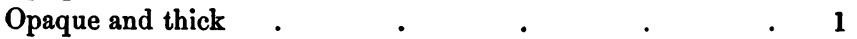

Covered by false membrane on its tympanal aspect $\quad 1$

Covered by fibrinous deposit on its cochlear aspect $\quad 1$

Auditory Nerve.

Red at its entrance into the vestibule $\quad$. $\quad . \quad$. 1

Garotid Canal.

Contracted

I would venture to call the attention of the Society to the very large proportion of cases in which disease of a decided character has been found. The ears have been taken indiscriminately from subjects of all ages, yet only 23 per cent. are healthy. Nor is it unimportant to observe that in two instances in which the tympanic cavity in children has been found in an advanced stage of inflammatory disease, there existed no symptoms during life by which special attention would have been directed to the ear.

And, in conclusion, I would express my concurrence in the opinion pronounced by Mr. Toynbee, that "investigations into the nature and treatment of diseases of the ear will be fruitful in valuable results if they are steadily pursued in an earnest and patient spirit." 